\title{
Training module on ultrasound during resuscitation
}

Domagoj Damjanovic ${ }^{1}$, Tobias Schroeder ${ }^{2}$, Carsten Lott ${ }^{3,4}$, Joerg Hanusch ${ }^{5}$, Caroline Busche ${ }^{6}$, Matthias Ott ${ }^{1}$, Christopher Starz ${ }^{7}$, Raoul Breitkreutz $^{8,9}$
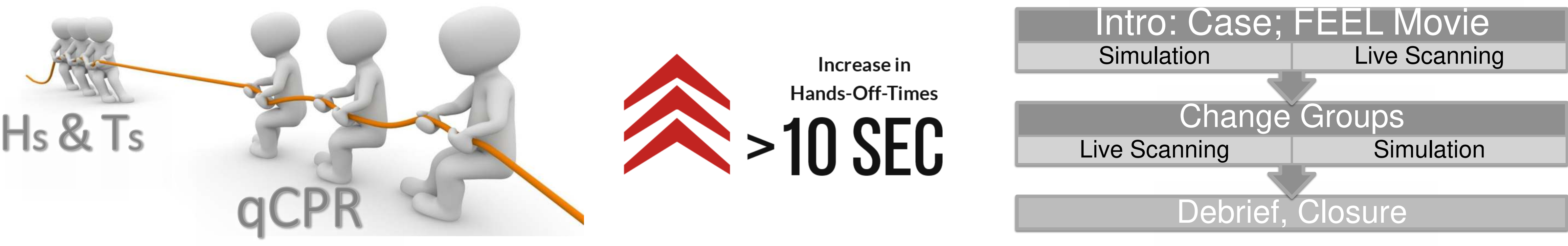

INTRODUCTION

Point of Care Ultrasound (PoCUS) during cardiopulmonary resuscitation ("Resuscitation Ultrasound") is increasingly available. It has a potential to identify reversible causes of cardiac arrest, guides invasive procedures and significantly alter patient management. It is part of the current universal ERC-Advanced Life Support-, ALS-algorithm. Recent research describes increased no-flow times due to use of ultrasound during resuscitation. Several authors therefore highlight the importance of an ALS-conformed integration of Resuscitation Ultrasound. ALS-conformed means to structure the procedure in order to minimize interruptions and use the chance to find reversible causes of cardiac arrest at an early point of time. We describe the development, implementation and one-year experience of a training module in Resuscitation Ultrasound, with a focus on procedural aspects .
Course evaluation during ERC Resuscitation 2017, N=35 (36)

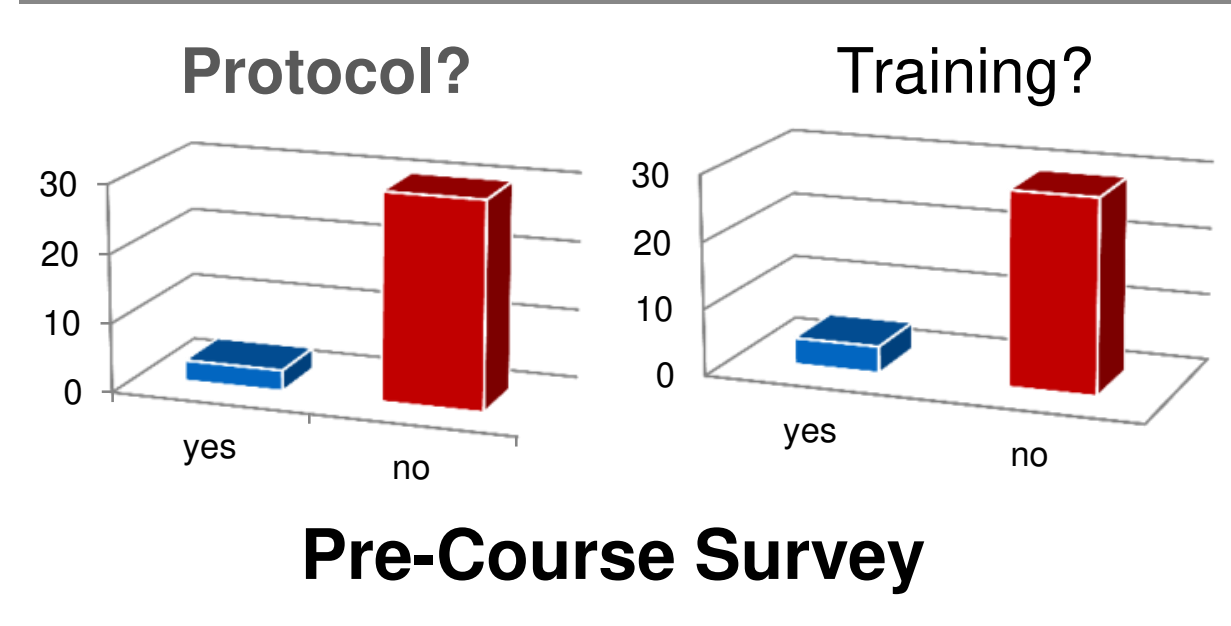

\section{METHODS}

Our working group developed a curriculum consisting of a brief introduction, live scanning on a volunteer (subxiphoid four-chamber heart view) and a simplified ALS scenario-training with a low-budget ultrasound simulation solution. The training module was piloted and adapted according to the evaluation.

\section{ACKNOWLEDGEMENTS}

Viola Sauter and Anne Bauer, Freiburg, for data management of course evaluation forms

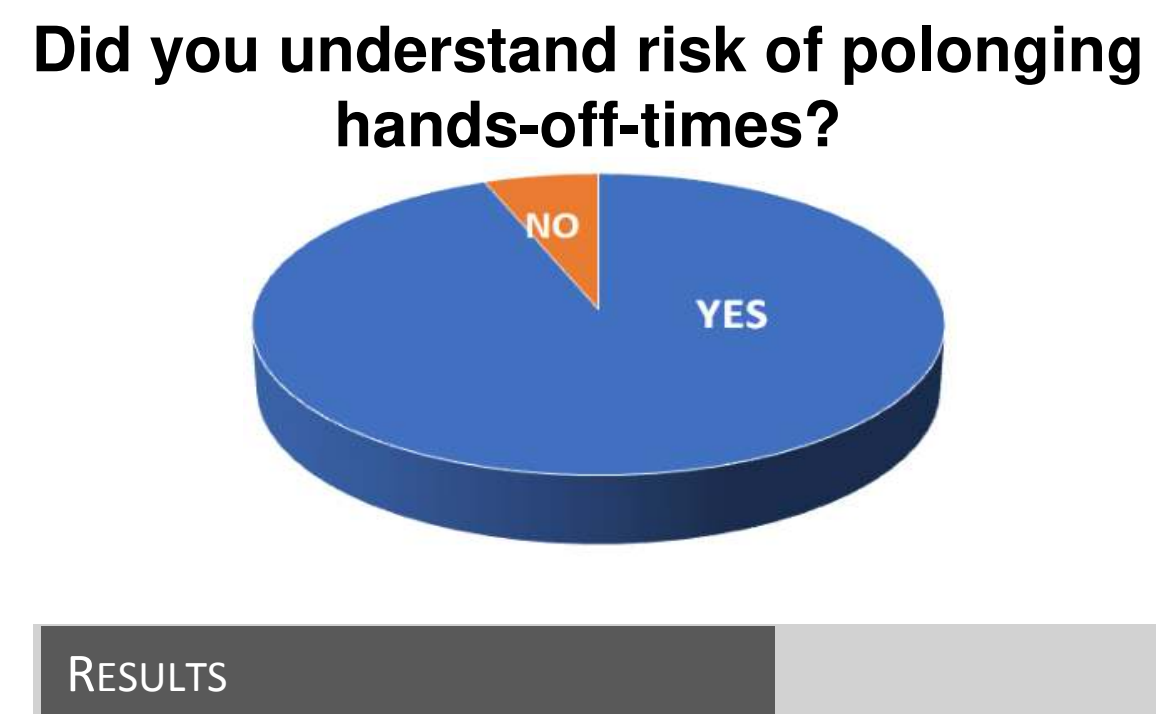

The module was first introduced during the ERC Congress 2017 in Freiburg, Germany. Since then, more than 200 attendees have been trained throughout the following months. The format underwent minor refinements, but it was broadly applicable and technically feasible within the given timeframe. Specific pitfalls of this training in ALS-conformed integration of Resuscitation Ultrasound have been identified that require further evaluation and can serve as training targets.

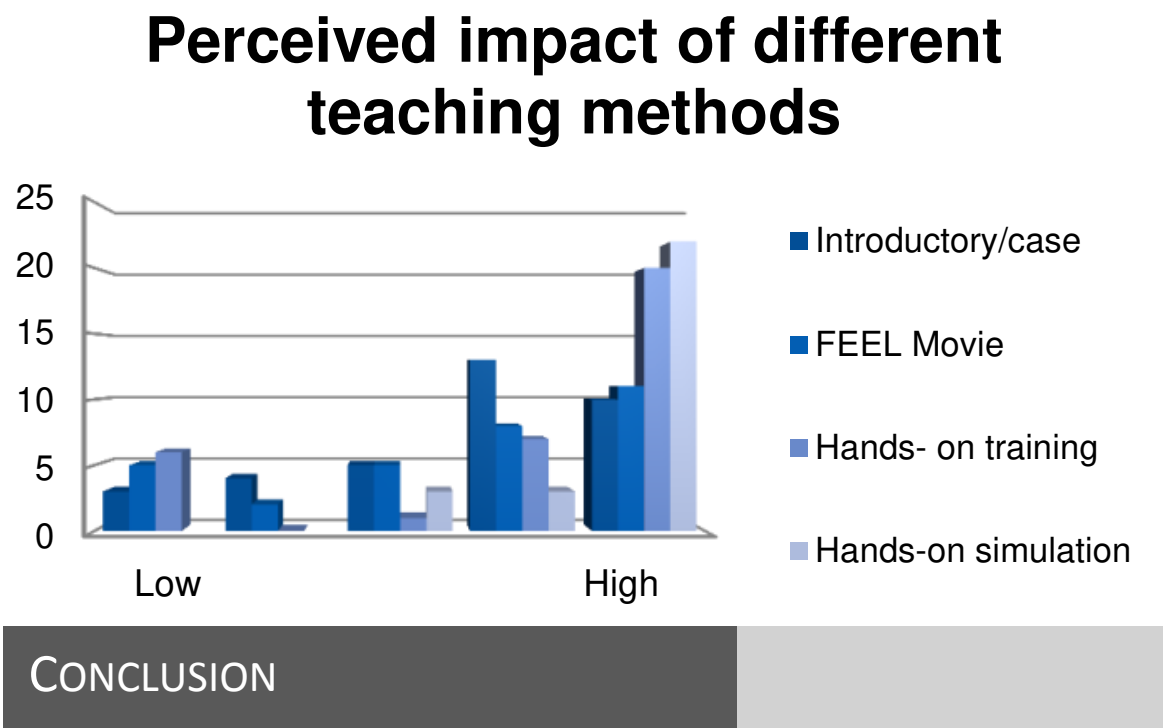

The training module on ALS-conformed Resuscitation Ultrasound has successfully been implemented. It has been designed for widespread adoption across training centers. Teaching experience suggests that ultrasound use significantly adds to complexity during ALS scenarios, and procedural training is warranted. Resource management and instructor qualification require further attention. Integration of ultrasound simulation technology is an important part of the didactic concept. Evaluation of the format is ongoing. 